\title{
EFFICACY PROFILE AND SAFETY OF VERY LOW DOSE RITUXIMAB IN PATIENTS WITH GRAVES' ORBITOPATHY
}

\author{
Guia Vannucchi ${ }^{1}$, Irene Campi ${ }^{1}$, Danila Covelli ${ }^{2}$, Nicola Currò ${ }^{3}$, Elisa Lazzaroni ${ }^{2}$, Andrea \\ Palomba ${ }^{4}$, Davide Soranna ${ }^{5}$, Antonella Zambon ${ }^{4}$, Laura Fugazzola ${ }^{1}$, Ilaria Muller ${ }^{2}$, Claudio \\ Guastella ${ }^{6}$, Mario Salvi $^{2}$
}

${ }^{1}$ Endocrine and Metabolic Department, Istituto Auxologico Italiano IRCCS, Milano; ${ }^{2}$ Departments of Clinical Sciences and Community Health, Graves' Orbitopathy Center, Endocrinology, ${ }^{3}$ Ophthalmology, Fondazione IRCCS Cà Granda, Milan, Italy; ${ }^{4}$ Department of Statistics and Quantitative Methods, Division of Biostatistics, Epidemiology and Public Health University of Milano-Bicocca, Milan - Italy - ${ }^{5}$ Istituto Auxologico Italiano, IRCCS, Milan, ${ }^{6}$ Otolaryngology, Fondazione IRCCS Cà Granda.

Short Title: Low dose rituximab in Graves' orbitopathy

Key words: Graves' disease, Graves' orbitopathy, Rituximab, CD 20, CAS, cytokine release syndrome, dysthyroid optic neuropathy,

Address for correspondence: Guia Vannucchi, MD, PhD

\author{
Endocrine and Metabolic Department, \\ Istituto Auxologico Italiano IRCCS, Milano \\ guiava@libero.it
}

This work was funded by: Fondazione IRCCS Cà Granda, Milan 
ABSTRACT

Background: Rituximab (RTX), a chimeric human-murine anti-CD20 monoclonal antibody, has been used for treatment of active moderate-severe Graves' orbitopathy (GO) since 2004 as second line therapy in patients unresponsive to intravenous steroids. We conducted an open label, prospective study (EUDRACT 2012-001980-53) in which patients were treated with a single infusion of only $100 \mathrm{mg}$ RTX to analyze the efficacy and safety of this low dose.

Methods: Seventeen patients, of whom nine had disease that was unresponsive to intravenous methylprednisolone, and eight with newly diagnosed GO were enrolled. Disease activity was assessed with the clinical activity score (CAS) and severity with a composite ophthalmic score. Long term surgical treatment and quality of life were also assessed, as well as treatment-related adverse events.

Results: Mean baseline CAS was $4.56 \pm 0.96$ and decreased to $1.25 \pm 1.14$ at 24 weeks $(P=0.001)$. Disease inactivation occurred within 24 weeks in $>90 \%$ of patients and was unrelated to disease duration. Severity improved in about $60 \%$ of patients, with no relapses. All patients showed peripheral depletion of $\mathrm{CD}_{2} \mathrm{O}^{+}$and $\mathrm{CD} 19^{+}$cells at the end of the RTX infusion (60 minutes). Two patients required surgical orbital decompression because of optic neuropathy (ON). Among adverse events observed, there was one patient who developed a cytokine release syndrome.

Conclusions: A dose of $100 \mathrm{mg}$ RTX is effective in patients with active moderate-severe GO. Low doses are better tolerated, expose patients to immune suppression for a shorter period of time and are extremely cost effective, compared to higher doses. This dose, consistently with all other immunosuppressants, does not prevent the progression of GO to dysthyroid optic neuropathy. 


\section{INTRODUCTION}

Graves' orbitopathy (GO), the most frequent extra-thyroidal manifestation of Graves' disease (GD), is a rare disorder that occurs in about $25-40 \%$ of patients with GD in a clinically relevant form (1) Intravenous glucocorticoids (IVGC) are considered the first line therapy for active moderate-severe GO (2); however, about $30 \%$ of patients are unresponsive or only temporarily responsive to steroids and therefore require second line immunosuppressive treatment. Among those proposed, rituximab (RTX), a chimeric human-murine anti-CD20 monoclonal antibody, has been used as off label treatment of active moderate-severe GO since 2004 (3) and, according to the EUGOGO guidelines, is the only second line therapy that does not require concurrent steroid administration. RTX has been approved for the treatment of several autoimmune diseases over the last few years, such as rheumatoid arthritis and ANCA related vasculitis (4). In GO, RTX has been shown to inactivate disease and to significantly modify its natural course, as suggested by the lack of disease reactivation shown in patients after treatment (5).

The dose of RTX initially employed in the treatment of active moderate-severe GO was based on previous work in autoimmunity (6) and consisted of $1000 \mathrm{mg}$ twice at two weeks interval (7-9). Due to the observation that in GO total peripheral (and orbital) B cell depletion was occurring even after low doses of RTX (100 mg) (10), we have been effectively reducing the therapeutic dose to a single infusion of $500 \mathrm{mg}$ RTX. This approach is justified by the results of a dose-finding analysis in a randomized trial comparing RTX to intravenous steroids (5) showing that $500 \mathrm{mg} \mathrm{RTX}$ induce inactivation of GO as effectively as $2 \times 1000 \mathrm{mg}$.

The advantages of using lower RTX doses are to expose patients to lower risks of potentially severe side effects (11-13), and to limit the cost ( $€ 300-600$ per patient) of the treatment, currently prescribed off label.

This open label prospective study was designed to analyze the efficacy, in terms of disease inactivation and improvement of severity, of a single $100 \mathrm{mg}$ RTX dose in a group of consecutive patients with active moderate-severe GO of different duration. The main objective of this study was to assess the cumulative evidence of the therapeutic benefit of RTX in GO versus the adverse events and safety implications. 


\section{PATIENTS AND METHODS}

\section{Patients}

Seventeen consecutive patients with active moderate-severe GO, 14 with GD and three with Hashimoto's thyroiditis (HT), 14 women and three men, aged 28-72 yr (mean \pm SD, 51.5 \pm 11.6$)$ were recruited between 2012 and 2015 and treated with one single $100 \mathrm{mg}$ dose of RTX. One woman with GD and active GO, treated according to the protocol, interrupted the infusion of RTX after only $25 \mathrm{mg}$, because of the onset of a rapid infusion reaction due to cytokine release (described below in detail). The duration of active disease (de novo or reactivated after previous immunosuppressive treatment discontinued for at least 3 months) ranged between 0.8-10.4 months (mean \pm SD 4.2 \pm 3.3 ). At the time of RTX therapy, 13 patients were euthyroid on methimazole (MMI) treatment, one was untreated because of GD remission, patient was euthyroid one year after radioiodine therapy, one had euthyroid Hashimoto's thyroiditis, while three patients were hypothyroid on Lthyroxine, of whom one after total thyroidectomy. The clinical characteristics of the patients are summarized in Table 1.

Patients were seen in a joint thyroid and eye clinic (Graves' Orbitopathy Center, Endocrinology, Milan) and at each visit blood was taken for thyroid function tests, serum autoantibody testing and peripheral blood lymphocyte count. Twelve of 17 patients received previous steroid treatment, while five RTX as first line therapy. Patients were assessed at baseline and at 4, 8, 12, 16, 24, 32, 40 and 76 weeks after treatment. The ophthalmological examination included lid fissure and Hertel measurements, color vision, cover test, Hess-Lancaster screen, visual acuity, tonometry, fundus examination and visual field. Disease activity was graded with the clinical activity score (CAS) (14). All patients had active $\mathrm{GO}$ as assessed by a baseline CAS $\geq 3 / 7$ or $>4 / 10$ points, at subsequent examinations. Orbital CT scan was performed only in patients who required the study of the optic nerve in suspected optic neuropathy.

\section{Therapeutic protocol}

Patients received rituximab (MabThera; Hoffman La Roche, Basel, Switzerland), according to the protocol, as a single dose of $100 \mathrm{mg}$ infused over approximately $1 \mathrm{hr}$ and $15 \mathrm{~min}$. One hour prior to RTX infusion oral paracetamol (1 gr), chlorphenamine $(10 \mathrm{mg})$ and i.v. 
hydrocortisone $(100 \mathrm{mg}$ ) were administered to prevent possible allergic reactions. Contraindications to RTX therapy were pregnancy, breast feeding, diabetes, known coronary artery disease, significant cardiac arrhythmias, severe congestive heart failure, serious chronic illness, active infection, a history of recurrent infection or recurrent bacterial infections, history of sarcoidosis, primary or secondary immunodeficiency, history of hypersensitivity, known anaphylaxis to mouse-derived proteins, positive PPD without documentation of treatment for TB infection, a history of cancer excluding resected basal or major squamous cell carcinoma, cervical dysplasia or in situ cervical cancer, denied consent to HIV testing, allergy to paracetamol, chlorphenamine, hydrocortisone (used for premedication). The study protocol was registered (EUDRACT 2012-001980-53) and approved by the Ethics Committee of the Fondazione IRCCS Cà Granda of Milan. All patients gave informed consent for participation in the trial.

\section{Biochemical and immunological testing}

Serum TSH, FT4 and FT3 concentrations and antithyroglobulin (TgAb) and antithyroperoxidase antibodies (TPOAb) levels were measured using the AutoDELFIA ${ }^{\mathrm{TM}}$ technique (Perkin Elmer ${ }^{\mathrm{TM}}$-Life Sciences, Wallac Oy, Turku, Finland). Serum TSH receptor antibodies (TRAb), detected as TSH binding inhibitory immunoglobulins, were measured using a 3rd generation TRAK test (BRAHMS TRAK human KRYPTOR (Thermo Fisher Scientific, Germany).

\section{Cytofluorimetric analysis}

We studied the pattern of peripheral blood lymphocytes before RTX and throughout the study period. We analyzed the standard immunophenotypic panel (CD3+, CD3+4+, $\mathrm{CD} 3+8+, \mathrm{CD} 3+\mathrm{DR}+, \mathrm{CD} 20+, \mathrm{CD} 19+5+, \mathrm{CD} 56+16+3)$ on aliquots of around $10^{5}$ lymphocytes, submitted to standard triple staining procedures in order to carry out immunogating with CD45, and the pairs of monoclonal antibodies to subpopulations of T, B and NK cells, subsequently processed in the flow cytometer (BD Facsan, Cell-quest software).

\section{Analysis of the therapeutic response}

The changes of activity and severity parameters after RTX were studied by assessing 1) the decrease of the CAS $\geq 2$ points compared to baseline (mourits); 2 ) the prevalence and time of disease inactivation (number of patients with CAS $<3$ ); 3 ) the disease improvement at 
24 weeks, by calculating a composite ophthalmic score (proposed by the EUGOGO guidelines) (2). Improvement was considered with a change in 2 or more of the following parameters, without deterioration of any of those in any of the eyes: (1) decrease of palpebral aperture of $\geq 3 \mathrm{~mm}$; (2) improvement in NOSPECS class II signs by $\geq 2$ grades; (3) decrease of one grade in the Bahn-Gorman diplopia score or improvement of $\geq 8^{\circ}$ in ductions; (4) reduction of proptosis of $\geq 2 \mathrm{~mm}$; (5) decrease of the CAS of $\geq 2$ points. Disease deterioration was assessed as development of dysthyroid optic neuropathy (DON) or occurrence of two of the following: (1) increase in palpebral aperture by at least $3 \mathrm{~mm}$; (2) increase of any of the class 2 signs of NOSPECS by at least 2 grades; (3) increase of proptosis by at least $2 \mathrm{~mm}$; (4) decrease of $\geq 8^{\circ}$ in motility or worsening of the diplopia score and (5) deterioration of the CAS. If changes were of a smaller magnitude than these parameters, the therapeutic outcome was considered as 'no change'.

\section{Quality of life}

The therapeutic outcome was also assessed by administering the disease specific quality of life questionnaire (GO-QoL) (15), for both patient's appearance and function at 24 weeks.

\section{Assessment of adverse events}

Treatment related side effects were recorded and assessed as serious and minor events during therapy and in the follow-up period. Side effects were classified as major (cytokine release syndrome, occurrence of major depression, severe infections) and minor (urticaria, allergic reactions-nose itching, sore-throat- fever, flushing, myalgia, asthenia, nausea). Side effects occurring during the infusions were usually controlled and monitored by the endocrinologist and did not influence the ophthalmological picture. The development of cytokine release syndrome, previously reported in GO patients treated with RTX was considered a serious adverse event. Disease progression to DON was not recorded as a side effect, but as ineffective treatment.

\section{Statistical analysis}

Sociodemographic and clinical continuous variables are expressed as means \pm standard deviation (SD) and categorical data as frequencies and proportions. An ANCOVA model for repeated measures was fitted to evaluate the trend of CAS values during the follow-up. To 
take into account the correlation among measurements on the same subject in different times a variance-covariance matrix with Toeplitz structure was considered. A statistical significance of a trend was evaluated by the $p$-value of the variable time included in the model. Other covariates considered in the model were: age, sex and disease duration. A nonparametric analysis of interval-censored data was carried out in order to estimate the cumulative proportion (and relative 95\% confidence interval) of the composite dichotomous endpoint. Patients undergoing surgical orbital decompression during the observation period, up to 24 weeks, were not included in the analysis of the disease outcomes.

\section{RESULTS}

\section{Analysis of efficacy on disease activity and severity}

Disease activity was assessed in all patients as a CAS of at least 4 points out of 10 . Mean baseline CAS was $4.56 \pm 0.96$ and decreased to $2.0 \pm 1.57$ at 12 weeks $(P=0.001)$, to $1.46 \pm 1.2$ at 16 weeks $(P=0.001)$, to $1.25 \pm 1.14$ at 24 weeks $(P=0.001)$ and to $1.4 \pm 1.26$ at 32 weeks $(P=0.001)$ (Figure $1 A)$. At 12 weeks, 13 of 15 patients had inactive $\mathrm{GO}(\mathrm{CAS}<3)(86.6 \%)$ one patient had a decrease of CAS from 7 to 5 and one worsened with an increase of CAS from 4 to 5 . We excluded one patient with unilateral GO who rapidly responded to RTX (4 weeks) and decided to undergo surgical orbital decompression before 12 weeks, for cosmetic reasons. The probability of cumulative inactivation rate after RTX was more than $50 \%$ after four weeks and increased to $93.8 \%$ (15 of 16 patients; $95 \% \mathrm{Cl}: 63.2 \%$ to $99.1 \%$ ) at week 12 without changes until week 40, as shown in Figure 1B. At 24 weeks all patients were inactive. None of the patients showed GO relapses at 52 and 76 weeks of follow-up. In order to study whether the duration of the disease might influence the therapeutic response to RTX, we analyzed disease inactivation in relation to the onset of active GO. Patients were divided into two groups based on the mean duration which was $4.2 \pm 3.3$ months (range 0.5-10.3 months). Patients with a longer disease duration responded to RTX as well as those with a short duration, and in fact after week 4 the responses of the former group were overall better $(p<0.0001)$ (Figure 2).

At 24 weeks severity improvement, assessed by the composite ophthalmic score, was observed in seven of 12 patients (58.3\%). In three patients GO remained stable and in two 
worsened (lid fissure and proptosis, proptosis and CAS respectively). Between 12 and 24 weeks from RTX treatment two patients developed DON, possibly present in a subclinical form and not fully recognized at baseline examination, as frequently occurs in GO. Another patient with inactive disease, underwent elective surgical orbital decompression for marked unilateral proptosis (Figure 3).

The degree of residual disease was studied at 76 weeks. Six patients did not require further treatments, five patients underwent elective surgical decompression. Seven and two patients underwent squint surgery and eye lid correction, respectively (Figure 3).

The GO-QoL questionnaire was completed by 14 patients. At 24 weeks the appearance score improved in eight patients, stabilized in two and worsened in four. The function score improved in seven patients, stabilized in four and worsened in three. The QoL appearance scale score was $>50 \%$ in 11 patients at baseline and in 13 patients at both 12 and 24 weeks, while the QoL function score was $>50 \%$ in 9 patients at baseline, in 10 at 12 weeks and in eight at 24 weeks.

\section{$B$ cell depletion after treatment}

All patients showed peripheral depletion of $\mathrm{CD} 2 \mathrm{O}^{+}$and $\mathrm{CD} 19^{+}$cells at the end of the RTX infusion (60 minutes). B cells began repopulating after 4 to 12 weeks, and at 40 and 76 weeks of follow-up we observed peripheral B cell return of nearly $53 \%$ and $70 \%$ (data not shown), respectively, compared to baseline. The CAS continued to decrease independently of $B$ cell repopulation.

\section{Effect on serum TSH-receptor antibodies}

We measured serum TRAb levels at each follow-up visit in all patients and we observed a progressive decrease of the antibodies at 12 weeks $(P=0.14)$ reaching significance only at 24 weeks $(P=0.015)$ (not shown).

\section{Side effects}

The treatment was well tolerated by the majority of patients. Minor side effects, such as sore-throat and nose itching usually associated with the infusion of higher dose RTX, were not seen with a dose of $100 \mathrm{mg}$ in this series of patients. One woman reported mild urticaria which resolved with low dose steroids. 
A 69-year old woman with contraindications to intravenous methylprednisolone therapy, due to previous steroid-induced pyelonephritis and subsequent nephrectomy, after administering $25 \mathrm{mg}$ of RTX showed rapidly progressive edema accompanied by orbital pain and decrease of vision in OD (5/10). She had active GO in OD, with chemosis, edema, plica and significant worsening of proptosis, with a CAS of 4/10 and inactive GO in OS. RTX was immediately withdrawn and $100 \mathrm{mg}$ hydrocortisone administered. Orbital pain improved over the next 15 minutes and visual acuity completely recovered in one hour without further treatment. Orbital CT imaging, carried out during amaurosis, ruled out optic nerve compression. A week later GO was inactive (CAS 3/10) and her vision was back to normal $(10 / 10)$. The patient's inflammatory signs and symptoms eventually resolved (CAS 0/10) with only residual proptosis and intermittent diplopia. Peripheral depletion of $\mathrm{CD}^{+} \mathrm{O}^{+}$and $\mathrm{CD} 19^{+}$cells was detected at $30 \mathrm{~min}$, after $25 \mathrm{mg}$ RTX (Figure 4). The patient's quality of life, showed significant improvement (57\%) of the functioning scale compared to baseline with resumption of daily activities.

\section{DISCUSSION}

The first and most important finding of this study is that a very low dose of RTX induces total peripheral B cell depletion and is rapidly effective for the treatment of active moderate-severe GO. Based on these results, we have evidence that a RTX dose (100 mg) even 20-fold lower than that commonly employed in other autoimmune disease may be used as a therapeutic option for GO (10), when first-line therapy fails (2). This study was designed to be open label and prospective with the aim to analyze the pattern of response and the profile of efficacy of RTX in relation to safety. The use of low dose RTX in GO may reduce the impact of generalized immunosuppression and protect the patients from the risks that may derive from prolonged B cell depletion (12).

One important observation, derived from comparison with higher RTX doses (1000 or 500 $\mathrm{mg}$ ), is that even at this low dose RTX inactivated GO very rapidly, within 12 weeks in $94 \%$ of patients, as shown by the analysis of cumulative rate of inactivation. In a recent retrospective study, Du Pasquier-Fediaevsky et al. (16) described similar results in patients with GO unresponsive to or relapsed after steroid treatment or external radiotherapy (RT). They reported a success rate of $87 \%$ with no relapses at 8 weeks, although all but two 
patients received at least two doses of $100 \mathrm{mg}$ of RTX. They did not observe differences in the efficacy of RTX in relation to the cumulative dose administered, but did not analyze the response in relation to the duration of GO. As we learned from the outcome of two randomized control trials of RTX in GO, the duration of the disease is likely a crucial factor that influences its effectiveness and the clinical response (17). For this reason, in the present series we analyzed response in relation to GO duration and found that patients with longer duration had a more consistent decrease of the CAS than those with shorter duration. There may be several explanations for this unexpected finding: 1) all patients in our study had a relatively short duration of the eye disease ( $<10$ months); 2 ) based on the dyphasic course of GO (Rundle curve), RTX treatment might accelerate the transition from the dynamic to the stable phase of the disease, especially in patients whose progression is close to the apex of the curve (18). Although unlikely, we cannot rule out also that GO became inactive due to the natural course of disease.

While the overall improvement of severity parameters was observed in about $60 \%$ of patients, none of the responders had relapse of GO at the end of follow-up, suggesting that RTX may modify the natural course of disease. The lack of disease reactivation was observed despite that B cells repopulated earlier (4-12 weeks) when compared to higher RTX doses (20-32 weeks) (5). It is important to underscore that RTX was the only modality of treatment of all but two patients, who subsequently required surgical orbital decompression after developing DON. This data is consistent with recent results published by Karasek et al. (19) who treated 10 patients with a single dose of $100 \mathrm{mg}$ RTX and reported DON in two patients. Similarly to our observations, in their cohort those two patients were steroid resistant and one with initial signs of DON at the time of treatment. Insull et al. (20) have used low dose (100 mg) RTX in GO patients in association to low dose steroids (mean dose $2.23 \mathrm{~g}$ ) or other immunosuppressive agents with the objective of reducing the dose of systemic steroids and increase the efficacy of therapy. They reported a $67 \%$ rate of disease inactivation and a $50 \%$ reduction of the severity score and no relapses at $>6$ months. They also observed progression to DON in three patients. The efficacy of combined immunosuppressive therapy needs to be verified in a randomized 
controlled trial. Furthermore, this approach might be accompanied by an increased risk of long term adverse events, especially infections (21).

Progression of moderate-severe GO to DON has been reported in patients treated with any of the currently employed immunosuppressive agents (25-27). Consistent with previous trials of RTX with either high dose (17) or low dose $(19,20)$, DON manifested also in two of the patients of the present study. Whether progression was due to unresponsiveness to RTX or to the presence of subclinical DON signs at the time of therapy is not clear, although by retrospectively reviewing these patients records we cannot rule out that they may have had suspected DON (19). These were the only two patients who required prompt additional treatment (decompressive surgery).

Rehabilitative surgery was required in about $50 \%$ of patients and in all of them was carried within 76 weeks from initial treatment, as the lack of disease relapse observed after RTX was reassuring for earlier surgical intervention in these patients. The patients' quality of life, especially visual functioning, was positively affected by low dose RTX, although the assessment of this parameters on a small series of patients may be of limited value. The observed decrease of serum TRAb levels may be due to a direct effect of RTX on TSH receptor stimulating antibodies in these patients $(25,26)$ or indirectly as a consequence of attained remission of hyperthyroidism after antithyroid treatment (27). This was not investigated specifically in this study.

By using low dose RTX we did not observe the common mild infusion-related side effects described when using 1000 mg RTX (5). Unfortunately, dose reduction did not prevent the onset of the syndrome of cytokine release that we observed in one patient after infusing as low as $25 \mathrm{mg}$ RTX. For this reason, we suggest that this treatment should only be administered in specialized centres, where all the appropriate rescue measures can be undertaken. This syndrome is characterized by acute orbital edema and some degree of visual loss which appears to be transient, followed by spontaneous recovery (10). A small dose of steroids has been used to control the acute event (28), but other drugs than steroids (i.e. tocilizumab) may be used for effectively treating the cytokine release syndrome (29). As shown by imaging, vision impairment was not caused by compression of the optic nerve at the orbital apex, suggesting a different mechanism. Venous outflow is 
important in the pathophysiological processes occurring in the orbit, and congestion of the superior orbital vein plays a key role also in the inflammatory phase of GO, as demonstrated by computed tomography and color doppler imaging studies $(30,31)$. The release of cytokines and the activation of the complement cascade after RTX may have caused transient impairment of the superior orbital vein flow, resulting in acute orbital vascular congestion, in marked edema of soft periorbital and intraorbital tissues (32), but only transient compressive effects on the optic nerve. There are also some reports suggesting that RTX may induce arterial vasospasm, which may cause transient ischemia of the optic nerve and contribute to determine the visual symptoms. Acute coronary vasospasm has been reported as infusion-related adverse effect of RTX (33) in a patient within 10 minutes from the first infusion, and retinal arteriolar spasm has been shown to cause transient monocular visual loss (34). The acute effect of RTX on the venous and arterial compartment may therefore explain the observed clinical picture, which is however transient and manageable in contrast with compressive DON, which requires urgent surgical orbital decompression.

The major limitation of this study is its uncontrolled design although, rather than comparing efficacy with standard steroid therapy for GO, the main objective was to analyze the timing of the therapeutic response to a small dose of RTX in relation to safety concerns in using this immunosuppressant. On the other hand, the study protocol was rigorous and gave us the opportunity to also treat a number of GO patients with a low dose of RTX as the only and first line therapy, which proved to be effective.

In conclusion, the results of this study suggest that low dose RTX is as effective as higher doses for the treatment for patients with active moderate-severe GO. Reduced RTX doses may be used in selected patients as first-line therapy instead of steroids. Besides being better tolerated, low dose RTX expose patients to B cell depletion and immune suppression for a shorter period of time, although, we show in this study that $100 \mathrm{mg}$ RTX, consistent with all other immunosuppressants, does not prevent the progression of GO to DON in predisposed patients. Another advantage for a single low-dose infusion of RTX is the reduction of hospital admissions and treatment costs, even if compared to standard intravenous steroid therapy. Currently the cost of $100 \mathrm{mg}$ RTX is about $€ 350$ per vial.

Disclosure Statement. The Authors have nothing to disclose. 


\section{REFERENCES}

1. Perros P, Hegedüs L, Bartalena L, Marcocci C, Kahaly G, Baldeschi L, Salvi M, Lazarus JH, Eckstein A, Pitz S, Boboridis K, Anagnostis P, Ayvaz G, Boschi A, Brix TH, Currò N, Konuk $O$, Marinò $M$, Mitchell AL, Stankovic B, Törüner $F B$, von Arx G, Zarković $M$ and Wiersinga WM 2017 Graves' orbitopathy as a rare disease in Europe: a European Group on Graves' Orbitopathy (EUGOGO) position statement. Orphanet Journal of Rare Diseases 12:72

2. Bartalena L, Baldeschi L, Boboridis K, Eckstein A, Kahaly GJ, Marcocci C, Perros P, Salvi M, Wiersinga WM; European_Group on Graves'Orbitopathy (EUGOGO). 2016 The 2016 European Thyroid Association G on Graves' Orbitopathy Guidelines for the Management of Graves' Orbitopathy. Eur Thyroid J 5: 9-26. doi: 10.1159/000443828.

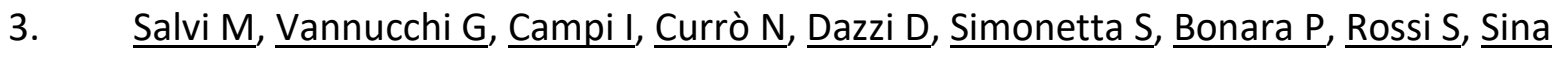
C, Guastella C, Ratiglia R, Beck-Peccoz P. 2007 Treatment of Graves' disease and associated ophthalmopathy with the anti-CD20 monoclonal antibody rituximab: an open study. Eur J Endocrinol 156: 33-40

4. Dorner T \& Lipsky PE 2014 B cells: depletion or functional modulation in rheumatic diseases. Current Opinion in Rheumatology 26: 228-236.

5. Salvi M, Vannucchi G, Currò N, Campi I, Covelli D, Dazzi D, Simonetta S, Guastella C, Pignataro L, Avignone S, Beck-Peccoz P 2015 Efficacy of B-cell targeted therapy with rituximab in patients with active moderate to severe Graves' orbitopathy: a randomized controlled study. J Clin Endocrinol Metab 100:422-31.

6. Edwards JC, Cambridge G \& Leandro MJ 2006 B cell depletion therapy in rheumatic disease. Best Practice and Research: Clin Rheumatol 20: 915-928.

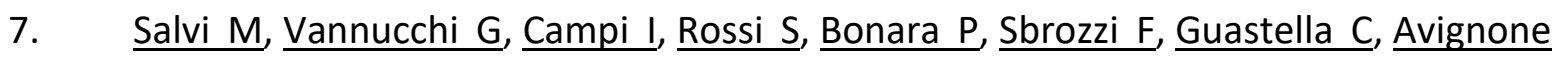
$\underline{\text { S}}, \underline{\text { Pirola G }}$, Ratiglia R, Beck-Peccoz P 2006 Efficacy of rituximab treatment for thyroidassociated ophthalmopathy as a result of intraorbital B-cell depletion in one patient unresponsive to steroid immunosuppression, Eur J Endocrinol 154: 511-517. 
8. Salvi M , Vannucchi G, Campi I, Currò N, Dazzi D, Simonetta S, Bonara P, Rossi S, Sina C, Guastella C, Ratiglia R, Beck-Peccoz P 2007 Treatment of Graves' disease and associated ophthalmopathy with the anti-CD 20 monoclonal antibody rituximab: an open study, Eur J Endocrinol 156: 33-40.

9. Khanna D, Chong KK, Afifiyan NF, Hwang CJ, Lee DK, Garneau HC, Goldberg RA, Darwin CH, Smith TJ, Douglas RS 2010 Rituximab Treatment of Patients with Severe, Corticosteroid-Resistant Thyroid-Associated Ophthalmopathy, Ophthalmology 117: 133139

10. Salvi $M$, Vannucchi $G$, Currò $N$, Introna $M$, Rossi $S$, Bonara $P$, Covelli $D$, Dazzi D, Guastella C, Pignataro L, Ratiglia R, Golay J, Beck-Peccoz 2012 P.A small dose of rituximab may be sufficient to treat Graves' orbitopathy: new insights into the mechanism of action. Arch Ophthalmol 130: 122-24

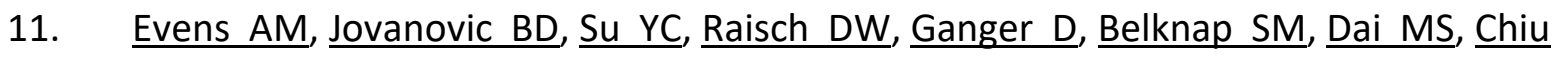
BC, Fintel B, Cheng Y , Chuang SS, Lee MY, Chen TY, Lin SF, Kuo CY 2011 Rituximabassociated hepatitis B virus (HBV) reactivation in lymphoproliferative diseases: metaanalysis and examination of FDA safety reports. Ann Oncol.22:1170-80

12. van Vollenhoven RF, Emery $\mathrm{P}$, Bingham CO 3rd, Keystone EC, Fleischmann RM, Furst DE, Tyson N, Collinson N, Lehane PB 2013 Long-term safety of rituximab in rheumatoid arthritis: 9.5-year follow-up of the global clinical trial programme with a focus on adverse events of interest in RA patients. Ann Rheum Dis 72:1496-502

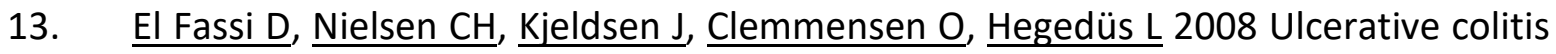
following B lymphocyte depletion with rituximab in a patient with Graves' disease. Gut 57:714-715.

14. Mourits MP, Prummel MF, Wiersinga WM, Koorneef L 1997 Clinical activity score as a guide in the management of patients with Graves' ophthalmopathy. Clin Endocrinol 47:914. 
15. Terwee C, Wakelkamp I, Tan S, Dekker F, Prummel MF, Wiersinga W 2002 Longterm effects of Graves' ophthalmopathy on health-related quality of life. Eur J Endocrinol 46:751-7.

16. Du Pasquier-Fediaevsky L, Andrei S, Berche M, Leenhardt L, Héron E, Rivière S 2018 Low-Dose Rituximab for Active Moderate-to-Severe Graves' Orbitopathy Resistant to Conventional Treatment. Ocul Immunol Inflamm 13:1-7.

17. Stan M \& Salvi M 2017 Rituximab therapy for Graves' orbitopathy - lessons from randomized control trials. Eur J Endocrinol 176: 101-R109

18. Campi I, Vannucchi G, Salvi M 2016 THERAPY OF ENDOCRINE DISEASE: Endocrine dilemma: management of Graves' orbitopathy. Eur J Endocrinol 175: R117-33. doi: 10.1530/EJE-15-1164.

19. Karasek D, Cibickova L, Karhanova M, Kalitova J, Schovanek J, Frysak Z 2017 Clinical and immunological changes in patients with active moderate-to-severe Graves' orbitopathy treated with very low-dose rituximab. Endokrynologia Polska 68:498-504.

20. Insull EA, Sipkova Z, David J, Turner He, Norris JH 2019 Early low-dose rituximab for active thyroid eye disease: an effective and well tolerated treatment. Clin Endocrinol 91:179-186

21. Kaegi C, Wuest B, Schreiner J, Steiner UC, Vultaggio A, Matucci A, Crowley C, Boyman O 2019 Systematic Review of Safety and Efficacy of Rituximab in Treating Immune-Mediated Disorders. Front Immunol 6:10:1990

22. Genere N, Stan MN 2019 Current and Emerging Treatment Strategies for Graves' Orbitopathy. Drugs 79:109-124. doi: 10.1007/s40265-018-1045-9. Review.

23. BartalenaL, Krassas GE, Wiersinga $W$, Marcocci $C$, Salvi $M$, Daumerie $C$, Bournaud C, Stahl M, Sassi L, Veronesi G 2012 Efficacy and Safety of Three Different Cumulative Doses of Intravenous Methylprednisolone for Moderate to Severe and Active Graves' Orbitopathy. J Clin Endocrinol Metab 97: 4454-4463, doi.org/10.1210/jc.2012$\underline{2389}$ 


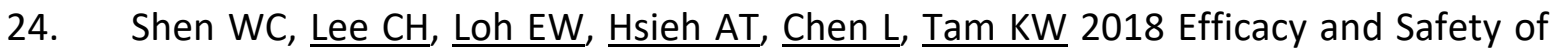
Rituximab for the Treatment of Graves' Orbitopathy: A Meta-analysis of Randomized Controlled Trials. Pharmacotherapy 38:503-510. doi: 10.1002/phar.2111.

25. Mitchell AL, Gan EH, Morris $M$, Johnson K, Neoh C, Dickinson AJ, Perros $\mathrm{P}, \underline{\text { Pearce }}$ SH 2013 The effect of B cell depletion therapy on anti-TSH receptor antibodies and clinical outcome in glucocorticoid refractory Graves' orbitopathy. Clin Endocrinol (Oxf) 79:437-42.

26. El Fassi D, Banga JP, Gilbert JA, Padoa C, Hegedüs L, Nielsen CH 2009 Treatment of Graves' disease with rituximab specifically reduces the production of thyroid stimulating autoantibodies. Clin Immunol 130:252-8.

27. Vannucchi G, Campi I, Bonomi M, Covelli D, Dazzi D, Currò N, Simonetta S, Bonara $\underline{\text { P }}$ Persani L, Guastella C, Wall J, Beck-Peccoz P, Salvi M 2010 Rituximab treatment in patients with active Graves' orbitopathy: effects on proinflammatory and humoral immune reactions, Clin Exp Immunol 161:436-43.

28. Fournier-Guilloux AL, Feigel-Guiller B, Lebranchu P 2016 Syndrome de relargage de cytokines avec réaction orbitaire aiguë au cours d'un traitement par rituximab pour orbitopathie basedowienne sévère : à propos d'un cas illustré. Annales d'Endocrinologie $\underline{77}$ : P 382

29. Karschnia P, Jordan JT, Forst DA, Arrillaga-Romany IC, Batchelor TT, Baehring JM, Clement NF, Gonzalez Castro LN, Herlopian A, Maus MV, Schwaiblmair MH, Soumerai JD, Takvorian RW, Hochberg EP, Barnes JA, Abramson JS, Frigault MJ, Dietrich J. Clinical presentation, management, and biomarkers of neurotoxicity after adoptive immunotherapy with CAR T cells. Blood. 2019 16;133(20):2212-2221. doi: 10.1182/blood2018-12-893396.

30. Alp MN, Ozgen A, Can I, Cakar P \& Gunalp I 2000 Colour Doppler imaging of the orbital vasculature in Graves' disease with computed tomographic correlation. $\mathrm{Br} \mathrm{J}$ Ophthalmol 84: 1027-30, doi: 10.1136/bjo.84.9.1027

31. Nakase Y, Osanai T, Yoshikawa K \& Inoue Y. Color Doppler imaging of orbital venous flow in dysthyroid optic neuropathy $1994 \mathrm{Jpn} J$ Ophthalmol 38: 80-6. 
32. Brunet-Possenti $F^{1}$, Franck N, Tamburini J, Jacobelli S, Avril MF, Dupin N 2011 Focal rituximab-induced edematous reaction at primary cutaneous follicle center lymphoma lesions: case report and literature review. Dermatology 223: 200-2 doi:

음
$10.1159 / 000332074$

33. Lee L, Ukreti V 2012 Rituximab-induced coronary vasospasm. Case Rep Hematol 984986. doi: 10.1155/2012/984986.

34. Stasi K, Ramchandran RS, Rao NA, Feldon SE, DiLoreto DA Jr 2009 Retinal arteriolar spasm during transient monocular visual loss in eosinophilic vasculitis. J Neuroophthalmol 29:58-61. doi: 10.1097/WNO.0b013e3181989ba8 
Table 1. Clinical, biochemical and immunological characteristics of patients at baseline.

\begin{tabular}{ll}
\hline Sex (female/male) & $13 / 3$ \\
Age (years) & $51.5 \pm 11.7$ \\
TSH (mU/L) & $1.4 \pm 1.6$ (n.v.0.28-4.3) \\
FT4 (pg/ml) & $12 \pm 3.6$ (n.v. 8-17) \\
FT3 (pg/ml) & $3.6 \pm 1.5$ (n.v. 2-5) \\
Primary thyroid dysfunction (n) & \\
Graves' disease & 13 \\
Hashimoto's thyroiditis & 3 \\
Serum TSH receptor antibodies (TRAb) & $27.6 \pm 42.8$ (n.v. <1.5) \\
CAS & $4.5 \pm 0.9$ \\
Lid fissure OD (mm) & $12.2 \pm 2.6$ \\
Lid fissure OS (mm) & $13 \pm 3$ \\
Hertel OD (mm) & $23.9 \pm 2.3$ \\
Hertel OS (mm) & $23.9 \pm 3.6$ \\
CD19 & $262.2 \pm 118$ (n.v. 100-500)
\end{tabular}

All values are expressed as mean \pm SD 


\section{FIGURE LEGENDS}

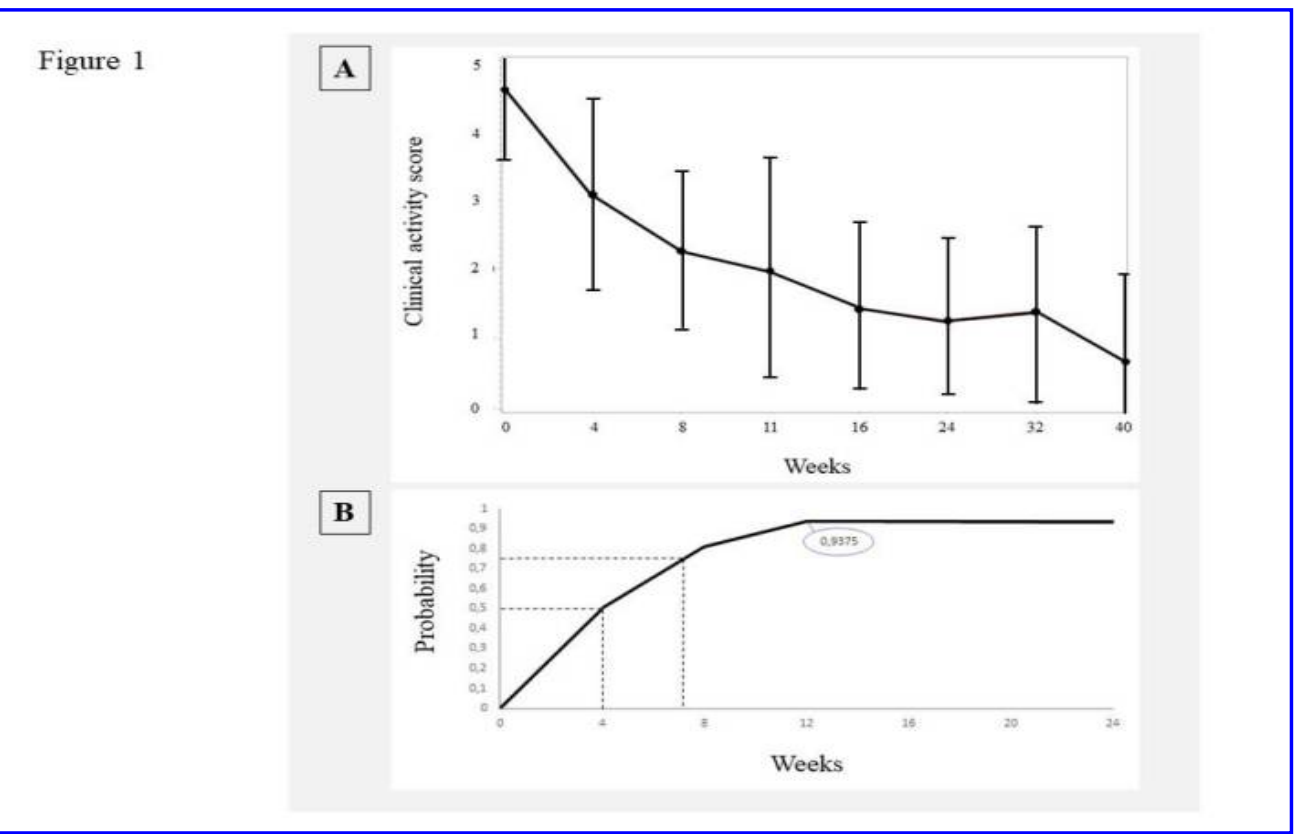

Figure 1

A) Decrease of the clinical activity score (CAS) in patients with moderate-severe Graves' orbitopathy after a single infusion of RTX $100 \mathrm{mg}$ from baseline to 40 weeks of follow-up $(P<0.001)$.

B) The probability of cumulative incidence rate of inactivation after low dose RTX: 50 $\%$ of patients at 4 weeks and $93 \%$ of patients at 12 weeks become inactive. 
Figure 2

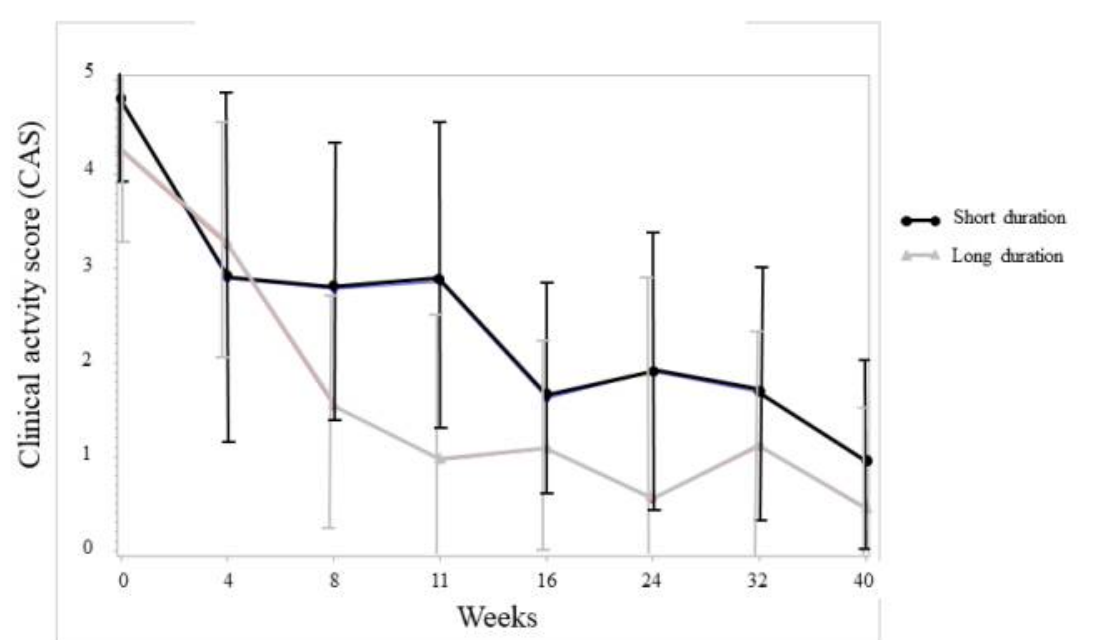

\section{Figure 2}

Decrease of the CAS in patients treated with a single $100 \mathrm{mg}$ of RTX in relation to the duration of Graves' orbitopathy assessed as less or more than the median duration in months (4.2 \pm 3.3 ; range 0.5-10.3 months). 
Figure 3

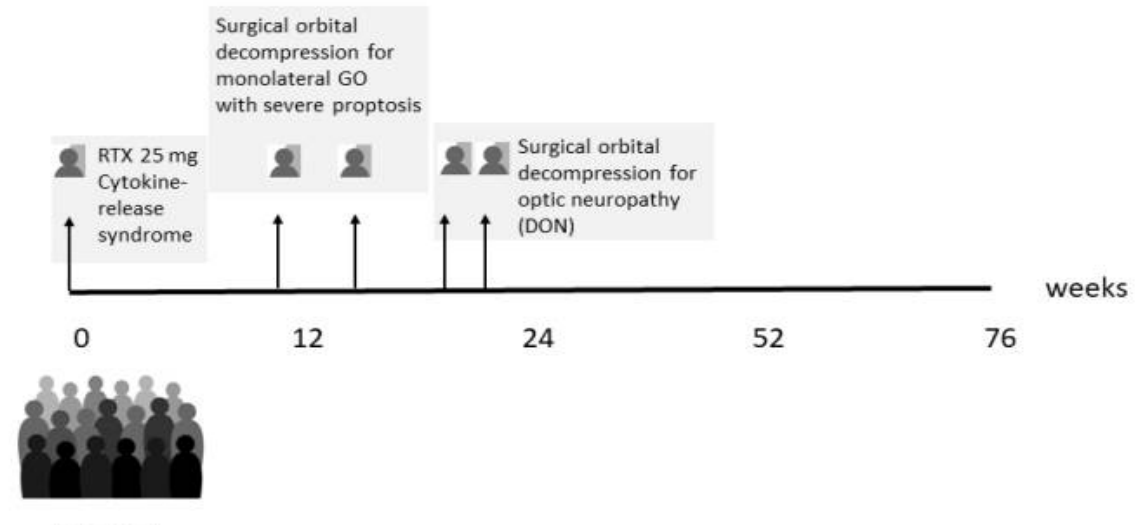

17 patients

\section{Figure 3}

Flow chart diagram of patients' included in the study. In detail, one patient only received $25 \mathrm{mg}$, two patients underwent early surgical orbital decompression (12-16 weeks) for monolateral marked proptosis and two patients underwent surgical orbital decompression for optic neuropathy (DON) between 12 and 24 weeks of follow-up. 


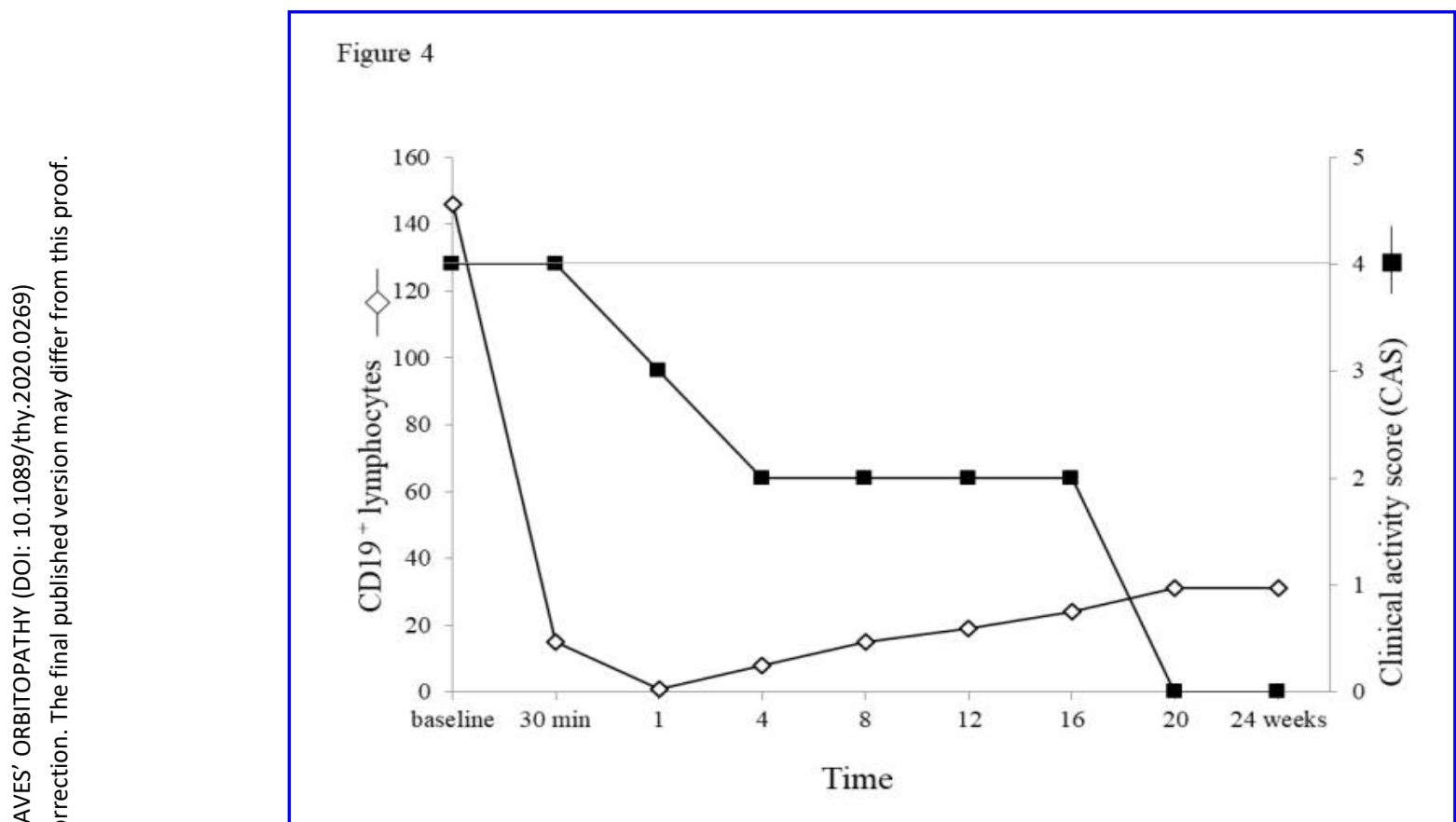

\section{Figure 4}

Total CD 19 depletion at 30 min after the infusion of $25 \mathrm{mg} \mathrm{RTX}$ in one patient developing a moderate syndrome of cytokine release and the subsequent decrease of the CAS without further therapeutic intervention. B cells begin repopulation around 4 weeks. 